\title{
On generalized Helmholtz type equations in concentric annular domains in $\mathbb{R}^{3}$
}

\author{
Denis Constales * Dennis Grob ${ }^{\dagger} \quad$ Rolf Sören Kraußhar ${ }^{\ddagger}$
}

October 30, 2008

\begin{abstract}
In this paper we consider inhomogeneous generalized Helmholtz type equations $\left(\Delta+\lambda^{2}\right) u=f$ in the annulus of an arbitrary ball in $\mathbb{R}^{3}$ with given boundary conditions, where we assume that $\lambda$ is an arbitrary complex number. Applying the hypercomplex operator calculus, one can express the solutions in terms of quaternionic integral operators. The quantitative entities to be determined in order to calculate these integral operators in practice are the Cauchy kernel and the Bergman kernel for eigensolutions to the Dirac operator in $\mathbb{R}^{3}$. In contrast to the Cauchy kernel which is universal for all domains in $\mathbb{R}^{3}$, the Bergman kernel however depends on the domain. In this paper we give an explicit formula for the Bergman kernel of the annulus of a ball in $\mathbb{R}^{3}$ with arbitrary radii $0<R_{1}<R_{2}<+\infty$ in terms of explicit special functions. With the knowledge of the Bergman kernel the appearing integral operators can be evaluated fully analytically and thus provide us with explicit formulas for the solutions.
\end{abstract}

Keywords: Helmholtz type equations, reproducing kernels, Bergman spaces, annular domains, quaternionic analysis

MSC Subject-Classification: 30 G 35; 32 A 25, 31 B 20.

\section{Introduction}

Let us consider the following concrete boundary value problem. Suppose that $\Omega$ is the annulus of a ball in $\mathbb{R}^{3}$ of radii $R_{1}, R_{2}$, i.e. $\Omega=\left\{\mathbf{z} \in \mathbb{R}^{3} \mid R_{1}<\|\mathbf{z}\|<R_{2}\right\}$ where $(0<$

\footnotetext{
*Department of Mathematical Analysis, Ghent University, Building S-22, Galglaan 2, B-9000 Ghent, Belgium. Financial support from BOF/GOA 01GA0405 of Ghent University gratefully acknowledged. Email: dc@cage. UGent.be

${ }^{\dagger}$ Department of Mathematics, Section of Analysis, Katholieke Universiteit Leuven, Celestijnenlaan 200B, B-3001 Leuven (Heverlee), Belgium. Financial support from FWO project G.0231.07 gratefully acknowledged. E-mail: dennisgrob@gmx. de

$¥$ Department of Mathematics, Section of Analysis, Katholieke Universiteit Leuven, Celestijnenlaan 200B, B-3001 Leuven (Heverlee), Belgium. Financial support from FWO project G.0335.08 gratefully acknowledged. E-mail: soeren.krausshar@wis.kuleuven.be
} 
$\left.R_{1}<R_{2}\right)$ and where $\|\cdot\|$ denotes the usual Euclidean norm. Let $f$ be a given function that is supposed to be an element of the Sobolev space $W^{2, k}(\Omega)$, i.e. its $k$-th derivative in the sense of Sobolev is square integrable over the annulus. Furthermore, suppose that $g$ is a given function on the boundary of the annulus belonging to $W^{2, k+3 / 2}(\partial \Omega)$. As shown for instance in [11] p. 81, in [13, 14, 16, 19] and in many other sources of literature the unique solution to the boundary value problem

$$
\begin{aligned}
\left(\Delta+\lambda^{2}\right) u(\mathbf{z}) & =f(\mathbf{z}) \text { on } \Omega \\
u(\mathbf{z}) & =g(\mathbf{z}) \text { at } \partial \Omega
\end{aligned}
$$

can be expressed in terms of quaternionic integral operators. If $\lambda$ is real, then we deal with the classical Helmholtz equation. The positive square root of $\lambda^{2}$ then has the physical interpretation as the wave number $k$. The solutions to the Helmholtz equation include the solutions to the time-harmonic Maxwell equations, see for instance $[13,14,16]$. If $\lambda$ is purely positively imaginary, say $\lambda=i \Lambda$ with $\Lambda>0$, then we deal with the Klein-Gordon equation in the time-independent case, making the identification $\Lambda=\frac{m c}{\hbar}$, where $m$ stands for the mass, $c$ for the speed of light and where $\hbar$ is the Planck number, cf. [15]. Here, an in all that follows variables that are set in bold face, such as $\mathbf{z}:=z_{1} e_{1}+z_{2} e_{2}+z_{3} e_{3}$ are vector variables from $\mathbb{R}^{3}$. As well-known, using the quaternionic calculus the above described system can be rewritten in the form

$$
\begin{aligned}
(D-\lambda)(D+\lambda) u(\mathbf{z}) & =f(\mathbf{z}) \text { on } \Omega \\
u(\mathbf{z}) & =g(\mathbf{z}) \text { at } \partial \Omega,
\end{aligned}
$$

where $\mathbf{D}:=\sum_{i=1}^{3} \frac{\partial}{\partial z_{i}} e_{i}$ is the Dirac operator, and where the elements $e_{1}, e_{2}, e_{3}$ of the standard basis from $\mathbb{R}^{3}$ which will be interpreted as the quaternionic imaginary units obeying the usual quaternionic multiplication rules as described in the preliminary section of this paper. We admit that the functions $f$ and $g$ may be scalar-valued, or vector-valued, real quaternionic-valued and even complex quaternionic-valued, too. Adapting the calculations from [11] pp.81-83 to the slightly more general framework of admitting complex values for $\lambda$, the solution to the posed boundary value problem can also be written as

$$
u=F_{\lambda} g+T_{-\lambda} \mathbf{P}_{\lambda}(\mathbf{D}-\lambda) h-T_{-\lambda}\left(I-\mathbf{P}_{\lambda}\right) T_{\lambda} f \in W^{2, k+2}(\Omega),
$$

where $h$ is an $W^{2, k+2}(\Omega)$-extension of $g$. Here $I$ is the identity operator, $T_{\lambda}$ the Teodorescu transform, $F_{\lambda}$ the Cauchy transform and $\mathbf{P}_{\lambda}$ the Bergman projection for the operator $\mathbf{D}-\lambda$ associated to the domain $\Omega$. The Teodorescu transform and the Cauchy transform are independent of the domain $\Omega$. In the 3-dimensional case they have the following universal representation

$$
\left(T_{\lambda} u\right)(\mathbf{z})=-\int_{\Omega} e_{\lambda}(\mathbf{z}-\mathbf{w}) u(\mathbf{w}) d V_{\mathbf{w}} \quad \mathbf{z} \in \mathbb{R}^{3}
$$

and

$$
\left(F_{\lambda} u\right)(\mathbf{z})=\int_{\partial \Omega} e_{\lambda}(\mathbf{z}-\mathbf{w}) n(\mathbf{w}) u(\mathbf{w}) d S_{\mathbf{w}} \quad \mathbf{z} \in \mathbb{R}^{3} \backslash \partial \Omega,
$$


respectively, where $n(\mathbf{w})$ stands for the exterior normal field at $\mathbf{w} \in \partial \Omega$ and where

$$
e_{\lambda}(\mathbf{z})= \begin{cases}\frac{1}{4 \pi} e^{i \lambda\|\mathbf{z}\|}\left(\frac{\lambda}{\|\mathbf{z}\|}-\frac{\mathbf{z}}{\|\mathbf{z}\|^{3}}(1-i \lambda\|\mathbf{z}\|)\right), & \Im(\lambda)>0 \\ \frac{1}{4 \pi} e^{-i \lambda\|\mathbf{z}\|}\left(\frac{\lambda}{\|\mathbf{z}\|}-\frac{\mathbf{z}}{\|\mathbf{z}\|^{3}}(1+i \lambda\|\mathbf{z}\|)\right), & \Im(\lambda)<0\end{cases}
$$

is the fundamental solution to $(\mathbf{D}-\lambda) u=0$ in $\mathbb{R}^{3}$. These formulas can be derived from the formulas given in [20] when adapting her formulas, containing Bessel functions, to the particular 3-dimensional case. In the limit case $\Im(\lambda) \rightarrow 0$ one obtains for real $\lambda \neq 0$ the representation

$$
e_{\lambda}(\mathbf{z})=-\frac{\mathbf{z}}{4 \pi\|\mathbf{z}\|^{3}}[\cos (\lambda\|\mathbf{z}\|)+\|\mathbf{z}\| \lambda \sin (\lambda\|\mathbf{z}\|)]+\frac{\lambda \cos (\lambda\|\mathbf{z}\|)}{4 \pi\|\mathbf{z}\|} e_{0},
$$

which is also given (up to a minus sign) in [11]. In the case $\lambda=0$ the expression $e_{\lambda}(\mathbf{z})$ reduces to the usual Cauchy kernel function, i.e. $-\frac{\mathbf{z}}{4 \pi\|\mathbf{z}\|^{3}}$.

The Bergman projection $\mathbf{P}$, however, is strongly dependent on the domain. The determination of an explicit formula for the involving Bergman kernel associated to the solutions to $(\mathbf{D}-\lambda) u=0$ is very difficult in general. In [2] an explicit representation formula for the Bergman kernel in the unit ball for the solutions of the special system $\left(\mathbf{D}_{\mathbf{z}}-\lambda\right) u=0$, for arbitrary $\lambda \in \mathbb{C}$, has been developed. J. Ryan showed in [17] that the space of solutions to $\left(\mathbf{D}_{\mathbf{z}}-\lambda\right) u=0$ that are square-integrable over a domain that has a piecewise $C^{1}$ or Lipschitz boundary, has in general always a uniquely defined Bergman kernel function. In [7] the authors gave an explicit formula for the Bergman kernel of the unit ball associated to the more general system

$$
\left(\mathbf{D}_{\mathbf{z}}-\lambda_{1}\right)\left(\mathbf{D}_{\mathbf{z}}-\lambda_{2}\right) \cdots\left(\mathbf{D}_{\mathbf{z}}-\lambda_{p}\right) u(\mathbf{z})=0,
$$

where $\lambda_{1}, \ldots, \lambda_{p}$ are mutually distinct arbitrary non-zero complex numbers. We also proved that the Bergman kernel does exist for any $p \in \mathbb{N}$ and for any arbitrary domain $\Omega \subset \mathbb{R}^{n}$.

In this paper we give an explicit formula for the Bergman kernel of the annulus of the three-dimensional ball of arbitrary radii $0<R_{1}<R_{2}$ for square integrable functions that satisfy inside that annulus the equation $(\mathbf{D}-\lambda) u=0$. This allows us to evaluate the Bergman projection explicitly and to evaluate the representation formula (1) for the solutions to the posed generalized inhomogeneous Helmholtz equation in such an annulus fully analytically.

In our forthcoming papers $[5,6]$ the techniques presented in this paper will be extended to the more general context of polynomial Dirac equations of arbitrary degree with arbitrary complex coefficients in annuli of the $n$-dimensional unit ball.

\section{Preliminaries}

Let $\left\{e_{1}, e_{2}, e_{3}\right\}$ be the standard basis of the Euclidean vector space $\mathbb{R}^{3}$. We embed this vector space into the four-dimensional space of quaternions $\mathbb{H}$ whose elements 
have the form $a=a_{0} e_{0}+\mathbf{a}$ with $\mathbf{z}=a_{1} e_{1}+a_{2} e_{2}+a_{3} e_{3}$ and where $e_{0}:=1$ is the neutral element concerning multiplication. In the quaternionic calculus one has the multiplication rules $e_{1} e_{2}=e_{3}=-e_{2} e_{1}, e_{2} e_{3}=e_{1}=-e_{3} e_{2}, e_{3} e_{1}=e_{2}=-e_{1} e_{3}$, and $e_{j} e_{0}=e_{0} e_{j}$ and $e_{j}^{2}=-1$ for all $j=1,2,3$. With this multiplication operation $\mathbb{H}$ is a skew field. The scalar part of a quaternion $a$ denoted by $\operatorname{Sc}(a)$, is defined as the $a_{0}$ term. Correspondingly, one defines the vector part of the quaternion $a$ as $\operatorname{Vec}(a):=a_{1} e_{1}+a_{2} e_{2}+a_{3} e_{3}=\mathbf{a}$.

The quaternionic conjugation on $a$ is defined by $\overline{e_{0}}=e_{0}$ and $\overline{e_{j}}=-e_{j}$ for $j=1,2,3$. The conjugated quaternion of $a$ thus has the form $\operatorname{Sc}(a)-\operatorname{Vec}(a)$.

By forming the tensor product $\mathbb{H} \otimes_{\mathbb{R}} \mathbb{C}$ we obtain the complexified quaternions, denoted by $\mathbb{H}(\mathbb{C})$. Its elements are represented in the form $\sum_{j=0}^{3} a_{j} e_{j}$ where the elements $a_{j}$ are complex numbers of the form $a_{j}=a_{j_{1}}+i a_{j_{2}}$. The complex imaginary unit $i$ commutes with all basis elements $e_{j}$, i.e. we have $i e_{j}=e_{j} i$ for all $j=0,1,2,3$. We denote the complex conjugate of a complex number $\lambda \in \mathbb{C}$ by $\lambda^{\sharp}$. For any elements $a \in \mathbb{H}(\mathbb{C})$ we have $(\bar{a})^{\sharp}=\overline{\left(a^{\sharp}\right)}$. On $\mathbb{H}(\mathbb{C})$ one considers a standard (pseudo)norm defined by $\|a\|=\left(\sum_{j=0}^{3}\left|a_{j}\right|^{2}\right)^{1 / 2}$. Here $|\cdot|$ is the usual absolute value of the complex number $a_{j}$.

Let $\Omega \subseteq \mathbb{R}^{3}$ be an open set and $\mathbf{z}:=z_{1} e_{1}++z_{2} e_{2}+z_{3} e_{3}$ be a vector variable. A real differentiable function $f: \Omega \rightarrow \mathbb{H}$ that satisfies inside of $\Omega$ the system $\mathbf{D}_{\mathbf{z}} f=0$ where $\mathbf{D}_{\mathbf{z}}=\sum_{j=1}^{3} \frac{\partial}{\partial z_{j}} e_{j}$ is called left monogenic with respect to the variable $\mathbf{z}$. The operator $\mathbf{D}_{\mathbf{z}}$ is the Euclidean Dirac operator. The associated function theory is often called quaternionic analysis. It provides a four-dimensional generalization of classical complex analysis and many powerful tools to treat higher dimensional boundary value problems from harmonic analysis, see for instance $[1,9,11]$. Applying the quaternionic calculation rules, the Dirac operator factorizes the Euclidean Laplacian viz $\mathbf{D}_{\mathbf{z}}^{2}=-\Delta_{\mathbf{z}}$.

Following for instance, $[2,11,14,17,20]$ and many others, a similar powerful function theory does also exist for functions that are in the kernel of $\mathbf{D}-\lambda$. In this setting, the functions take values in the complex quaternions. The set of functions that are squareintegrable solutions to $(\mathbf{D}-\lambda) u=0$ is a right Hilbert module and is often called the Bergman space of $\lambda$-holomorphic functions, see for instance [12]. The associated Bergman space is equipped with the quaternion-valued inner product of the form

$$
\langle f, g\rangle=\int_{\Omega} \overline{f(\mathbf{z})} \sharp g(\mathbf{z}) d x_{1} d x_{2} d x_{3}
$$

and the derived norm has the form

$$
\|f\|_{L^{2}}=\sqrt{\operatorname{Sc}\langle f, f\rangle}
$$

Following our previous work [7], for each domain $\Omega$ this function space has a reproducing kernel, called the Bergman kernel denoted by $B(\mathbf{z}, \mathbf{w})$. The associated projection

$$
\mathbf{P}[u(\mathbf{z})]:=\int_{\Omega} B(\mathbf{z}, \mathbf{w}) u(\mathbf{w}) d V_{\mathbf{w}},
$$


is an ortho-projection from $L^{2}(\Omega)$ into $L^{2}(\Omega) \cap \operatorname{Ker}(\mathbf{D}-\lambda)$. Following for instance [11], the space $L^{2}(\Omega)$ admits the direct orthogonal decomposition

$$
L^{2}(\Omega)=L^{2}(\Omega) \cap \operatorname{Ker}(\mathbf{D}-\lambda) \oplus L^{2}(\Omega) \cap \mathbf{D} \stackrel{\circ}{W}^{2, k}(\Omega)
$$

where $\stackrel{\circ}{W}^{2, k}(\Omega)$ is the subset of the Sobolev space $W^{2, k}(\Omega)$ that consists of functions which vanish at the boundary of $\Omega$. The aim of this paper is to give an explicit formula for the $\lambda$-holomorphic quaternionic Bergman kernel for the annular domain $\Omega=\{\mathbf{z} \in$ $\left.\mathbb{R}^{3} \mid R_{1}<\|z\|<R_{2}\right\}$ where $0<R_{1}<R_{2}<+\infty$. This then allows us to compute the ortho projectors in the solution formula (1) explicitly.

\section{Local representations of eigensolutions to the Dirac equation}

Basic tools for all that follows are the following local representation theorems for eigenfunctions of the Dirac operator with a non-zero complex eigenvalue, cf. [20]:

Lemma 1 (Taylor series expansion). Let $u$ be a $\mathbb{H}(\mathbb{C})$-valued function that satisfies in the 3-dimensional open ball $B\left(0, R_{2}\right):=\left\{\mathbf{z} \in \mathbb{R}^{3} \mid 0<\|\mathbf{z}\|<R_{2}\right\}$ the differential equation $\left(\mathbf{D}_{\mathbf{z}}-\lambda\right) u(\mathbf{z})=0$ for a complex parameter $\lambda \in \mathbb{C} \backslash\{0\}$. Then there exists a sequence of spherical monogenics of total degree $q=0,1,2, \ldots$, say $P_{q}(\mathbf{z})$, such that in each open ball $B\left(0, r_{2}\right)$ with $0<r_{2}<R_{2}$

$$
u(\mathbf{z})=\sum_{q=0}^{+\infty}\|\mathbf{z}\|^{-q-1 / 2}\left(J_{q+1 / 2}(\lambda\|\mathbf{z}\|)-\frac{\mathbf{z}}{\|\mathbf{z}\|} J_{q+3 / 2}(\lambda\|\mathbf{z}\|)\right) P_{q}(\mathbf{z}) .
$$

Here, $J$ denote the usual Bessel functions of the first kind with complex argument, for its definition see for instance see [10].

The spherical monogenics $P_{q}$ appearing in this representation are homogeneous monogenic polynomials of total degree $q$. They have the form

$$
P_{q}(\mathbf{x})=\sum_{q_{2}+q_{3}=q} V_{q_{2}, q_{3}}(\mathbf{x}) a_{q_{2}, q_{3}}
$$

where $q_{q_{2}, q_{3}}$ are constant quaternions and where $V_{q_{2}, q_{3}}$ stand for the Fueter polynomials. The latter ones have in the vector formalism the representation

$$
V_{q_{2}, q_{3}}(\mathbf{x}):=\frac{1}{|\mathbf{q}| !} \sum\left(x_{\sigma(1)}+x_{1} e_{1} e_{\sigma(1)}\right) \ldots\left(x_{\sigma(|\mathbf{q}|)}+x_{1} e_{1} e_{\sigma(|\mathbf{q}|)}\right)
$$

where $|\mathbf{q}|:=q_{2}+q_{3}$ and $\sigma(i) \in\{2,3\}$. The summation is extended over all distinguishable permutations of the expressions $\left(x_{\sigma(i)}+x_{1} e_{1} e_{\sigma(i)}\right)$ without repetitions.

Let us now consider functions that are eigensolutions to the Dirac equation in an annular domain of the form $B\left(0, R_{1}, R_{2}\right):=\left\{\mathbf{z} \in \mathbb{R}^{3} \mid R_{1}<\|\mathbf{z}\|<R_{2}\right\}$ where $R_{1}$ and $R_{2}$ are reals $0<R_{1}<R_{2}<+\infty$. In annular domains, the analogue of the local representation in Lemma 1 is the following Laurent expansion representation, cf. [20]: 
Lemma 2 (Laurent series expansion). Let $0<R_{1}<R_{2}<+\infty$. Let $u$ be a $\mathbb{H}(\mathbb{C})$-valued function that satisfies in the 3-dimensional annulus $B\left(0, R_{1}, R_{2}\right)$ the differential equation $\left(\mathbf{D}_{\mathbf{z}}-\lambda\right) u(\mathbf{z})=0$ for a complex parameter $\lambda \in \mathbb{C} \backslash\{0\}$. Then there exist two sequences of spherical monogenics of total degree $q=0,1,2, \ldots$, say $P_{q}(\mathbf{z})$ and $P^{\prime}{ }_{q}(\mathbf{z})$, such that in each annulus $B\left(0, r_{1}, r_{2}\right)$ with $0<R_{1}<r_{1}<r_{2}<R_{2}$

$$
\begin{aligned}
u(\mathbf{z}) & =\sum_{q=0}^{+\infty}\|\mathbf{z}\|^{-q-1 / 2}\left(J_{q+1 / 2}(\lambda\|\mathbf{z}\|)-\frac{\mathbf{z}}{\|\mathbf{z}\|} J_{q+3 / 2}(\lambda\|\mathbf{z}\|)\right) P_{q}(\mathbf{z}) \\
& +\sum_{q^{\prime}=0}^{+\infty}\|\mathbf{z}\|^{-q^{\prime}-1 / 2}\left(Y_{q^{\prime}+1 / 2}(\lambda\|\mathbf{z}\|)-\frac{\mathbf{z}}{\|\mathbf{z}\|} Y_{q^{\prime}+3 / 2}(\lambda\|\mathbf{z}\|)\right) P_{q^{\prime}}^{\prime}(\mathbf{z}) .
\end{aligned}
$$

Here, $Y$ denote the usual Bessel functions of the second kind with complex argument.

For the proof of Lemma 1 and Lemma 2, we refer the reader for instance to [20]. For the sake of readability, we introduce the notation $S_{q}(\mathbf{z}, \mathbf{w})$ for the Szegö kernel for $\mathbf{D}_{\mathbf{z}}$-monogenic homogeneous polynomials of total degree $q$ in the 3-dimensional unit ball $B(0,1)$, which equals

$$
S_{q}(\mathbf{z}, \mathbf{w})=\frac{(-1)^{q}}{4 \pi} \sum_{m=0}^{q}\left(\begin{array}{c}
-1 / 2+m \\
m
\end{array}\right)\left(\begin{array}{c}
1 / 2+(q-m) \\
q-m
\end{array}\right)(\mathbf{z w})^{m}(\mathbf{w} \mathbf{z})^{q-m} .
$$

\section{The Bergman kernel of the annulus for $\mathrm{D}-\lambda$}

In this section we present our main result:

Theorem 1 Let $\lambda \in \mathbb{C} \backslash\{0\}$ and let $R_{1}, R_{2}$ be two positive reals with $0<R_{1}<R_{2}<+\infty$. Define the expressions $f$ and $g$ by

$$
f_{q, \lambda}(\mathbf{z}):=\|\mathbf{z}\|^{-q-\frac{1}{2}}\left(J_{q+\frac{1}{2}}(\lambda\|\mathbf{z}\|)-\frac{\mathbf{z}}{\|\mathbf{z}\|} J_{q+\frac{3}{2}}(\lambda\|\mathbf{z}\|)\right)
$$

and

$$
g_{q, \lambda}(\mathbf{z}):=\|\mathbf{z}\|^{-q-\frac{1}{2}}\left(Y_{q+\frac{1}{2}}(\lambda\|\mathbf{z}\|)-\frac{\mathbf{z}}{\|\mathbf{z}\|} Y_{q+\frac{3}{2}}(\lambda\|\mathbf{z}\|)\right),
$$

where $J$ denotes the usual Bessel function of first kind and $Y$ the Bessel function of second kind. The Bergman kernel of the annulus $B\left(0, R_{1}, R_{2}\right)$ associated to the equation $\left(\mathbf{D}_{\mathbf{z}}-\lambda\right) u=$ 0 is then given by

$$
B_{R_{1}, R_{2} ; \lambda}(\mathbf{z}, \mathbf{w})=\sum_{q=0}^{\infty}\left(\left(f_{q, \lambda}(\mathbf{z}), g_{q, \lambda}(\mathbf{z})\right) S_{q}(\mathbf{z}, \mathbf{w})\left(\begin{array}{cc}
a_{q} & b_{q} \\
c_{q} & d_{q}
\end{array}\right)^{-1}\left(\begin{array}{c}
\bar{f}_{q, \lambda^{\sharp}}(\mathbf{w}) \\
\bar{g}_{q, \lambda^{\sharp}}(\mathbf{w})
\end{array}\right)\right) .
$$


Here, the matrix entries have the form

$$
\begin{aligned}
a_{q} & =\int_{R_{1}}^{R_{2}} r^{2 q+2} S c\left\{\bar{f}_{q, \lambda \sharp}(r \omega) f_{q, \lambda}(r \omega)\right\} d r \\
b_{q} & =\int_{R_{1}}^{R_{2}} r^{2 q+2} S c\left\{\bar{f}_{q, \lambda \sharp}(r \omega) g_{q, \lambda}(r \omega)\right\} d r \\
c_{q} & =\int_{R_{1}}^{R_{2}} r^{2 q+2} S c\left\{\bar{g}_{q, \lambda \sharp}(r \omega) f_{q, \lambda}(r \omega)\right\} d r \\
d_{q} & =\int_{R_{1}}^{R_{2}} r^{2 q+2} S c\left\{\bar{g}_{q, \lambda \sharp}(r \omega) g_{q, \lambda}(r \omega)\right\} d r .
\end{aligned}
$$

where we put $r:=\|\mathbf{w}\|$ and $\omega:=\frac{\mathbf{w}}{r} \in S:=\left\{\mathbf{z} \in \mathbb{R}^{3} \mid\|\mathbf{z}\|=1\right\}$.

Remark: The elements $a_{q}, b_{q}, c_{q}, d_{q}$ are actually constants and do not depend on $\omega$. Notice that

$$
S c\left\{\bar{f}_{q, \lambda^{\sharp}}(r \omega) f_{q^{\prime}, \lambda}(r \omega)\right\}=r^{-q-q^{\prime}-1}\left(J_{q+\frac{1}{2}}\left(r \lambda^{\sharp}\right) J_{q^{\prime}+\frac{1}{2}}(r \lambda)+J_{q+\frac{3}{2}}\left(r \lambda^{\sharp}\right) J_{q^{\prime}+\frac{3}{2}}(r \lambda)\right) .
$$

This expression is obviously independent of $\omega \in S$. For the sake of simplicity, in the following calculations we write for $a_{q}, b_{q}, c_{q}, d_{q}$ simply $a, b, c, d$.

Proof of Theorem 1. Let us set $\theta:=\operatorname{det}\left(\begin{array}{ll}a & b \\ c & d\end{array}\right)=a d-b c$. We have

$$
\begin{aligned}
& \left\langle B_{R_{1}, R_{2} ; \lambda}(\mathbf{z}, \mathbf{w}), f_{q^{\prime}, \lambda}(\mathbf{w}) S_{q^{\prime}}(\mathbf{w}, \mathbf{v})\right\rangle \\
= & \frac{1}{\theta} \int_{\mathbf{w} \in B\left(0, R_{1}, R_{2}\right)}\left(S _ { q , } ( \mathbf { z } , \mathbf { w } ) \left(f_{q, \lambda}(\mathbf{z}) d \bar{f}_{q, \lambda \sharp}(\mathbf{w}) f_{q^{\prime}, \lambda}(\mathbf{w})+g_{q, \lambda}(\mathbf{z})(-c) \bar{f}_{q, \lambda \sharp}(\mathbf{w}) f_{q^{\prime}, \lambda}(\mathbf{w})\right.\right. \\
& \left.\left.+f_{q, \lambda}(\mathbf{z})(-b) \bar{g}_{q, \lambda^{\sharp}}(\mathbf{w}) f_{q^{\prime}, \lambda}(\mathbf{w})+g_{q, \lambda}(\mathbf{z}) a \bar{g}_{q, \lambda}(\mathbf{w}) f_{q^{\prime}, \lambda}(\mathbf{w})\right) S_{q^{\prime}}(\mathbf{w}, \mathbf{v})\right) d V_{\mathbf{w}} .
\end{aligned}
$$

We first consider the expression

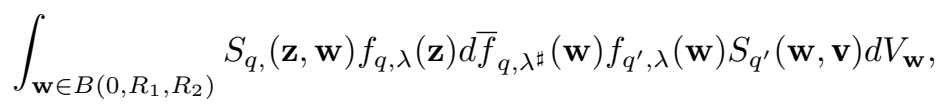

and represent it in polar coordinates:

$$
\int_{R_{1}}^{R_{2}} \int_{\omega \in S} S_{q},(\mathbf{z}, r \omega) f_{q, \lambda}(\mathbf{z}) d \bar{f}_{q, \lambda \sharp}(r \omega) f_{q^{\prime}, \lambda}(r \omega) S_{q^{\prime}}(r \omega, \mathbf{v}) d S_{\omega} r^{2} d r,
$$

where again $S:=\left\{\mathbf{z} \in \mathbb{R}^{3} \mid\|\mathbf{z}\|=1\right\}$ is the unit sphere in $\mathbb{R}^{3}$. Then we decompose the middle part of the integrand into its scalar and vector part:

$$
\bar{f}_{q, \lambda \sharp}(r \omega) f_{q^{\prime}, \lambda}(r \omega)=S c\left\{\bar{f}_{q, \lambda \sharp}(r \omega) f_{q^{\prime}, \lambda}(r \omega)\right\}+h(r) \omega .
$$


The term $\omega S_{q^{\prime}}(r \omega, \mathbf{v})$ is an outer spherical monogenic on $\omega \in S$. It is hence orthogonal to the expression $S_{q}(r \omega, \mathbf{v})$. Consequently, the contribution of $h(r) \omega$ in the integral vanishes. Only the expression

$$
\int_{R_{1}}^{R_{2}} \int_{\omega \in S} S_{q,}(\mathbf{z}, r \omega) f_{q, \lambda}(\mathbf{z}) d S c\left\{\bar{f}_{q, \lambda \sharp}(r \omega) f_{q^{\prime}, \lambda}(r \omega)\right\} S_{q^{\prime}}(r \mathbf{w}, \mathbf{v}) d S_{\omega} r^{2} d r,
$$

remains. As mentioned at the beginning the expression

$$
S c\left\{\bar{f}_{q, \lambda^{\sharp}}(r \omega) f_{q^{\prime}, \lambda}(r \omega)\right\}=r^{-q-q^{\prime}-1}\left(J_{q+\frac{1}{2}}\left(r \lambda^{\sharp}\right) J_{q^{\prime}+\frac{1}{2}}(r \lambda)+J_{q+\frac{3}{2}}\left(r \lambda^{\sharp}\right) J_{q^{\prime}+\frac{3}{2}}(r \lambda)\right) .
$$

is independent of $\omega \in S$. Therefore, the previous integral can be written as

$$
\int_{R_{1}}^{R_{2}} S c\left\{\bar{f}_{q, \lambda^{\sharp}}(r \omega) f_{q^{\prime}, \lambda}(r \omega)\right\} f_{q, \lambda}(\mathbf{z}) d\left(\int_{\omega \in S} S_{q}(\mathbf{z}, r \omega) S_{q^{\prime}}(r \omega, \mathbf{v}) d S_{\omega}\right) r^{2} d r .
$$

As a consequence of the reproduction property of $S_{q}(\mathbf{z}, \mathbf{w})$ and the homogeneity property $S_{q}(\mathbf{z}, r \omega)=r^{q} S_{q}(\mathbf{z}, \omega)$ which is valid for all real $r$, we can also write (6) in the form

$$
\delta_{q, q^{\prime}} d f_{q, \lambda}(\mathbf{z}) S_{q}(\mathbf{z}, \mathbf{v}) \int_{R_{1}}^{R_{2}} S c\left\{\bar{f}_{q, \lambda \sharp}(r \omega) f_{q^{\prime}, \lambda}(r \omega)\right\} r^{q+q^{\prime}+2} d r
$$

so that we obtain

$$
\begin{aligned}
& \sum_{q=0}^{\infty} \delta_{q, q^{\prime}} f_{q, \lambda}(\mathbf{z}) d S_{q^{\prime}}(\mathbf{z}, \mathbf{v}) \int_{R_{1}}^{R_{2}} S c\left\{\bar{f}_{q, \lambda^{\sharp}}(r \omega) f_{q^{\prime}, \lambda}(r \omega)\right\} r^{q+q^{\prime}+2} d r \\
= & f_{q, \lambda}(\mathbf{z}) d S_{q^{\prime}}(\mathbf{z}, \mathbf{v}) \int_{R_{1}}^{R_{2}} S c\left\{\bar{f}_{q^{\prime}, \lambda^{\sharp}}(r \omega) f_{q^{\prime}, \lambda}(r \omega)\right\} r^{2 q^{\prime}+2} d r
\end{aligned}
$$

which in turn equals

$$
\begin{aligned}
& f_{q, \lambda}(\mathbf{z}) a S_{q^{\prime}}(\mathbf{z}, \mathbf{v}) \int_{R_{1}}^{R_{2}}\left(J_{q^{\prime}+\frac{1}{2}}\left(r \lambda^{\sharp}\right) J_{q^{\prime}+\frac{1}{2}}(r \lambda)+J_{q^{\prime}+\frac{3}{2}}\left(r \lambda^{\sharp}\right) J_{q^{\prime}+\frac{3}{2}}(r \lambda)\right) r d r \\
= & a d f_{q, \lambda}(z) S_{q^{\prime}}(\mathbf{z}, \mathbf{v}) .
\end{aligned}
$$

Applying the same calculations to the remaining three summands, and summing up again over $q$, then we obtain

$$
\frac{1}{\theta}(a d-a c-b c+a c) f_{q, \lambda}(\mathbf{z}) S_{q^{\prime}}(\mathbf{z}, \mathbf{v})=\frac{1}{\theta} \operatorname{det}(\mathcal{M}) f_{q, \lambda}(\mathbf{z}) S_{q^{\prime}}(\mathbf{z}, \mathbf{v})=f_{q, \lambda}(\mathbf{z}) S_{q^{\prime}}(\mathbf{z}, \mathbf{v}) .
$$

The reproduction property now follows from the fact that the functions $f_{q, \lambda} S_{q^{\prime}}$ and $g_{q, \lambda} S_{q^{\prime}}$ form a generating system for the Bergman space.

It remains to show the invertibility of $\left(\begin{array}{ll}a & b \\ c & d\end{array}\right)$. Let $\left(\alpha_{1}, \alpha_{2}\right)^{t} \in \mathbb{C}^{2}$ be such that

$$
\left(\begin{array}{ll}
a & b \\
c & d
\end{array}\right)\left(\begin{array}{l}
\alpha_{1} \\
\alpha_{2}
\end{array}\right)=0
$$


Consider the function

$$
h:=f_{q, \lambda} \alpha_{1} S_{q}(\mathbf{z}, \mathbf{w})+g_{q, \lambda} \alpha_{2} S_{q}(\mathbf{z}, \mathbf{w}) .
$$

We have

$$
\begin{aligned}
\left\langle f_{q, \lambda} S_{q}(\mathbf{z}, \mathbf{w}), h\right\rangle & =\left\langle f_{q, \lambda} S_{q}(\mathbf{z}, \mathbf{w}), f_{q, \lambda_{j}} \alpha_{1} S_{q}(\mathbf{z}, \mathbf{w})+g_{q, \lambda} \alpha_{2} S_{q}(\mathbf{z}, \mathbf{w})\right\rangle \\
& =\alpha_{1}\left\langle f_{q, \lambda} S_{q}(\mathbf{z}, \mathbf{w}), f_{q, \lambda} S_{q}(\mathbf{z}, \mathbf{w})\right\rangle+\alpha_{2}\left\langle f_{q, \lambda} S_{q}(\mathbf{z}, \mathbf{w}), g_{q, \lambda} S_{q}(\mathbf{z}, \mathbf{w})\right\rangle \\
& =a \alpha_{1} S_{q}(\mathbf{z}, \mathbf{w})+b \alpha_{2} S_{q}(\mathbf{z}, \mathbf{w}) \\
& =(\underbrace{a \alpha_{1}+b \alpha_{2}}_{=0}) S_{q}(\mathbf{z}, \mathbf{w})=0 .
\end{aligned}
$$

Analogously we obtain

$$
\left\langle g_{q, \lambda} S_{q}(\mathbf{z}, \mathbf{w}), h\right\rangle=c \alpha_{j} S_{q}(\mathbf{z}, \mathbf{w})+d \alpha_{j} S_{q}(\mathbf{z}, \mathbf{w})=0, \quad \text { for } j=1,2 .
$$

So, we have

$$
h \perp f_{q, \lambda}, g_{q, \lambda},
$$

where the orthogonality relation has to be understood in the sense of the $L^{2}$-product involving the volume integral over annulus of the unit ball. In turn, from (7) we obtain

$$
h \perp\left(f_{q, \lambda} \alpha_{1} S_{q}(\mathbf{z}, \mathbf{w})+g_{q, \lambda} \alpha_{2} S_{q}(\mathbf{z}, \mathbf{w})\right),
$$

so $h \perp h$ and hence $h=0$. Since $S_{q} \neq 0$, it must hold that

$$
f_{q, \lambda} \alpha_{1}+g_{q, \lambda} \alpha_{2}=0 .
$$

Since the functions $\left\{f_{q, \lambda}, g_{q, \lambda}\right\}$ are linearly independent, it necessarily follows that $\alpha_{1}=\alpha_{2}=0$. Hence, the matrix $\left(\begin{array}{ll}a & b \\ c & d\end{array}\right)$ is invertible.

Remark: Let us finally consider the limit case $\lambda \rightarrow 0$ in Lemma 2. Then the Laurent series expression simplifies to the usual one for monogenic functions [8] which is of the form

$$
f(\mathbf{z})=\sum_{q=0}^{+\infty} P_{q}(\mathbf{z})+\sum_{q^{\prime}=0}^{+\infty} \frac{\mathbf{z}}{\|\mathbf{z}\|^{3+2 q}} P_{q^{\prime}}^{\prime}(\mathbf{z}) .
$$

In this case one replaces in Theorem 1 the expressions $f_{q, 0}$ by 1 and $g_{q, 0}$ by $\frac{\mathbf{z}}{\|\mathbf{z}\|^{3+2 q}}$ and we get the simplified formula

Theorem 2 The Bergman kernel of the annulus $B\left(0, R_{1}, R_{2}\right)$ associated to the equation $\mathbf{D}_{\mathbf{z}} u=0$ is then given by

$$
B_{R_{1}, R_{2} ; 0}(\mathbf{z}, \mathbf{w})=\sum_{q=0}^{\infty}\left(\left(1, \frac{\mathbf{z}}{\|\mathbf{z}\|^{3+2 q}}\right) S_{q}(\mathbf{z}, \mathbf{w})\left(\begin{array}{cc}
a_{q} & 0 \\
0 & d_{q}
\end{array}\right)^{-1}\left(\begin{array}{c}
1 \\
\|\mathbf{w}\|^{3+2 q}
\end{array}\right)\right) .
$$


Here, the matrix entries have the form

$$
\begin{aligned}
& a_{q}=\int_{R_{1}}^{R_{2}} r^{2 q+2} d r=\frac{\left(R_{2}^{2 q+1}-R_{1}^{2 q+1}\right)}{2 q+3} \\
& d_{q}=-\int_{R_{1}}^{R_{2}} r^{-2 q-2} d r=\frac{\left(R_{2}^{-2 q-1}-\mathbb{R}_{1}^{-2 q-1}\right)}{2 q+1} .
\end{aligned}
$$

where we put $r:=\|\mathbf{w}\|$.

In the case of the annulus of the unit ball, i.e. $\left.R_{1}=: \mu \in\right] 0,1\left[\right.$ and $R_{2}=1$ this formula simplifies to

$$
B_{\mu, 1 ; 0}(\mathbf{z}, \mathbf{w})=\sum_{q=0}^{\infty}\left(\left(1, \frac{\mathbf{z}}{\|\mathbf{z}\|^{3+2 q}}\right) S_{q}(\mathbf{z}, \mathbf{w})\left(\begin{array}{cc}
\frac{2 q+3}{\left(1-\mu^{2 q+1}\right)} & 0 \\
0 & \frac{2 q+1}{1-\mu^{-2 q-1}}
\end{array}\right)\left(\begin{array}{c}
1 \\
\frac{\mathbf{w}}{\|\mathbf{w}\|^{3+2 q}}
\end{array}\right)\right) .
$$

In view of the uniqueness of the Bergman kernel the representation (9) is equivalent to the following representation formula

$$
B_{\mu, 1 ; 0}(\mathbf{z}, \mathbf{w})=\frac{1}{4 \pi} \sum_{k \in \mathbb{Z}} \mathbf{D}_{z} \frac{\mu^{k}}{\left\|1+\mu^{2 k} \mathbf{z w}\right\|} \mathbf{D}_{w}
$$

that we worked out in our previous paper [4], which resulted by the application of the reflection principle from harmonic analysis. This formula in turn provides a direct analogy to the formula worked out by D. Calderbank for the Szegö kernel of nullsolutions to the equation $\mathbf{D}_{z} u=0$ in the annulus of the unit ball.

\section{References}

[1] F. Brackx, R. Delanghe and F. Sommen. Clifford Analysis. Pitman Res. Notes 76, BostonLondon-Melbourne, 1982.

[2] F. Brackx, F. Sommen, and N. Van Acker: Reproducing Bergman kernels in Clifford Analysis, Complex Variables 24 (1994), pp. 191 - 204.

[3] D. Calderbank: Dirac operators and Clifford analysis on manifolds with boundary. Max Planck Institute for Mathematics Bonn, Preprint number, 1996, pp. 96 - 131.

[4] D. Constales, D. Grob and R.S. Kraußhar: Explicit formulas for the Green's function and the Bergman kernel for monogenic functions in annular shaped domains in $\mathbb{R}^{n+1}$, to appear.

[5] D. Constales, D. Grob and R.S. Kraußhar: Reproducing kernel functions of solutions to polynomial Dirac equations in the annulus of the unit ball in $\mathbb{R}^{n}$ and applications to boundary value problems, in preparation.

[6] D. Constales, D. Grob and R.S. Kraußhar: Dirichlet type problems of polynomial Dirac equations with boundary conditions, in preparation.

[7] D. Constales, R.S. Kraußhar, Hilbert Spaces of Solutions to Polynomial Dirac equations, Fourier Transforms and Reproducing Kernel Functions for Cylindrical Domains, Zeitschrift für Analysis und Ihre Anwendungen 24 No. 3 (2005), pp. 611 - 636. 
[8] Delanghe, R., Sommen, F. and V. Souček: Clifford Algebra and Spinor Valued Functions, Dordrecht-Boston-London: Kluwer 1992.

[9] K. Gürlebeck, K. Habetha, and W. Sprößig: Funktionentheorie in der Ebene und im Raum. Birkhäuser Verlag, Basel, 2006

[10] I. Gradshteyn, and I.M. Ryzhik: Table of Integrals, Series and Products. New York: Academic Press 1980.

[11] K. Gürlebeck and W. Sprößig: Quaternionic analysis and elliptic boundary value problems, Basel: Birkhäuser, 1990.

[12] Kravchenko, V. and M. Shapiro: Helmholtz operator with a quaternionic wave number and associated function theory. In: Ławrynowicz, J. (ed.), Deformations of mathematical structures 2: Hurwitz-type structures and applications to surface physics. Selected papers from the seminar on deformations, Lódz-Malinka, Poland, 1988-1992, Dordrecht: Kluwer 1994, 101 - 128.

[13] V. Kravchenko and M. Shapiro, Helmholtz operator with a quaternionic wave number and associated function theory. II. Integral representations. Acta Applicandae Mathematicae 32 No. 3 (1993), pp. 243 - 265.

[14] V. Kravchenko and M. Shapiro: Integral representations for spatial models of mathematical physics, Harlow: Addison Wesley Longman 1996.

[15] V.V. Kravchenko, and P. R. Castillo: On the kernel of the Klein-Gordon operator. Z. Anal. Anwendungen 17 No. 2 (1998), pp. 261-265

[16] M. Mitrea: Boundary value problems and Hardy spaces associated to the Helmholtz equation in Lipschitz domains. J. Math. Anal. Appl. 202 No. 3 (1996), pp. 819 - 842.

[17] J. Ryan: Cauchy-Green type formulae in Clifford analysis. Trans. Am. Math. Soc. 347 No. 4 (1995), pp. 1331 - 1341.

[18] M. Shapiro and N. Vasilevski: On the Bergmann kernel function in hyperholomorphic analysis. Acta Appl. Math. 46 No. 1 (1997),pp. 1 - 27.

[19] W. Sprößig, Quaternionic analysis and Maxwell's equations. Cubo Mat. Educ. 7 No. 2 (2005), pp. $57-67$

[20] Zhenyuan Xu: A function theory for the operator $(D-\lambda)$, Complex Variables 16 No. 1 (1991), pp. $27-42$. 